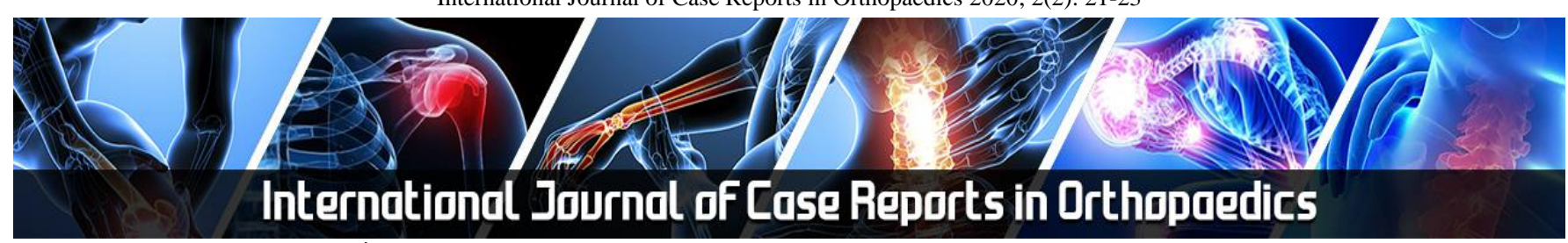

E-ISSN: 2707-8353 P-ISSN: 2707-8345 IJCRO 2020; 2(2): 21-23

Received: 21-05-2020 Accepted: 23-06-2020

\section{Sophia Burns}

MRCS, Registrar at North

East Thames (UCLH)

Rotation- Trauma and

Orthopaedics, the Whittington

Hospital, Magdala Avenue

London, N19 5NF

United Kingdom

\section{Peter Domos}

MD, FRCS, Tr and Ortho, Consultant Orthopaedic Surgeon Barnet General Hospital Well House Lane London, EN5 3DJ,

United Kingdom
Corresponding Author: Sophia Burns

MRCS, Registrar at North East Thames (UCLH) Rotation- Trauma and Orthopaedics, the Whittington Hospital, Magdala Avenue London, N19 5NF

United Kingdom

\section{Anterior fracture dislocation of proximal humerus with anatomical neck fracture: Case report and review of literature}

\section{Sophia Burns and Peter Domos}

DOI: https://doi.org/10.22271/27078345.2020.v2.i2a.24

\section{Abstract}

Fracture dislocation of the anatomical neck of humerus is a rare injury. The surgical management is guided by fracture morphology and displacement, potential disruption to blood supply, bone quality and the patient's functional demands. We have described a case of anterior fracture dislocation of the proximal humerus at the anatomical neck, managed successfully with cemented fracture-specific hemiarthroplasty. Through presentation of this rare case and discussion of the relevant literature, we hope to improve understanding of these injuries and the evidence base upon which to base their management.

Keywords: Humerus, fracture, dislocation, anatomical neck, hemiarthroplasty

\section{Introduction}

Fracture dislocation of the anatomical neck of humerus is an extremely rare injury. Of those reported the majority are posterior dislocations ${ }^{[1]}$. The management and long term outcomes following this injury are not well established; percutaneous closed reduction, open reduction internal fixation (ORIF) and hemiarthroplasty have all been described in the literature ${ }^{[1-6]}$.

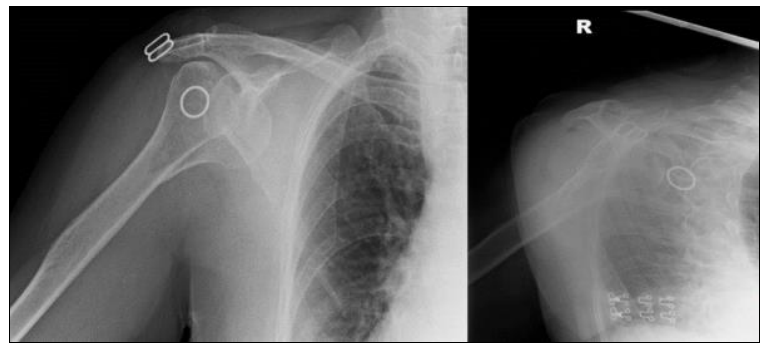

Fig 1: Antero-posterior (AP) and lateral Y- views of the right shoulder demonstrating 2-part anterior fracture dislocation of the proximal humerus

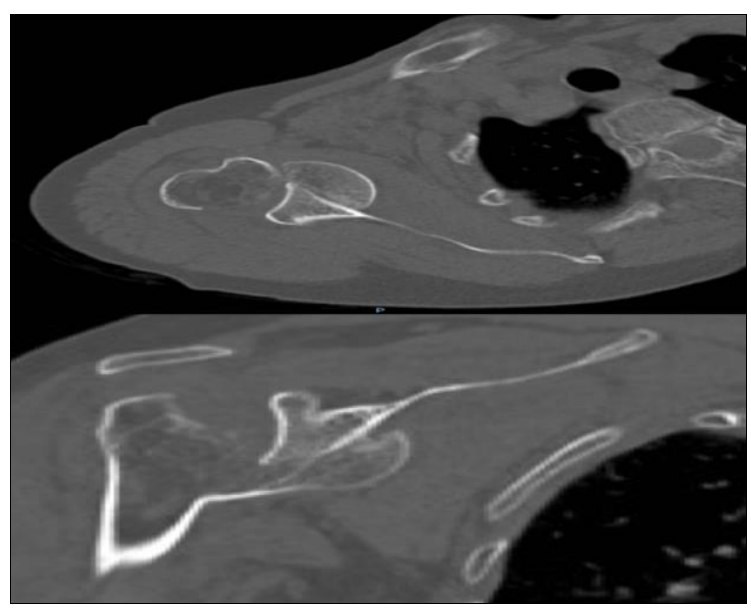

Fig 2: Axial and coronal slice from computed tomography (CT) scan at the level of the glenohumeral joint, demonstrating locked anterior dislocation of the humeral articular surface 


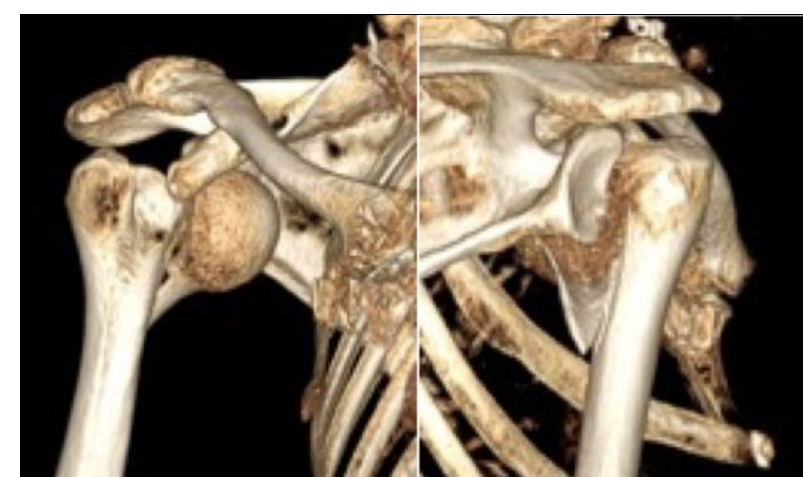

Fig 3: 3D reconstruction from CT demonstrating locked anterior dislocation of humeral articular surface

An emergent open reduction was not performed. Management options were discussed with the patient and her relatives and the decision was made to proceed with a planned surgery, to attempt an open reduction internal fixation but if not possible due to bone quality to change the plan to perform a hemiarthroplasty.

The patient underwent surgery on day 7 after the injury. A standard beach-chair position and deltopectoral approach was used to access the shoulder joint. The fracture was identified and first the humeral head was removed from the locked sub-coracoid position with care for the brachial plexus and subclavian vessels. The lesser and greater tuberosities were not fractured and the rotator cuff tendons were intact with age-related cuff changes. Due to poor bone quality and high risk for failed ORIF we decided to proceed with the hemiarthroplasty. After subscapularis tenotomy the medial calcar spike (which had very attenuated and minimal capsular attachments) was separated from the head fragment and reconstructed to the humeral shaft with 3 PDS-loop sutures using the Nice-knot technique. A UN displaced glenoid rim fracture was noted intra-operatively, this involved less than $20 \%$ of the anterior glenoid however the labrum remained intact, thus the decision was taken to leave this fracture as it was stable.

After standard preparation of the humeral shaft, the definitive head (size $39 \mathrm{~mm} \times 14$ ) and size 6.5 fracturespecific humeral stem (with central bone graft from humeral head) were assembled and implanted with bone cement (Wright Medical, Memphis, TN, USA). The intraoperative radiographs were satisfactory. After subscapularis repair the Nice-knot technique with 3 PDS-loop sutures was used to further repair and reinforce supraspinatus, subscapularis and infraspinatus to the proximal humerus and prosthesis.

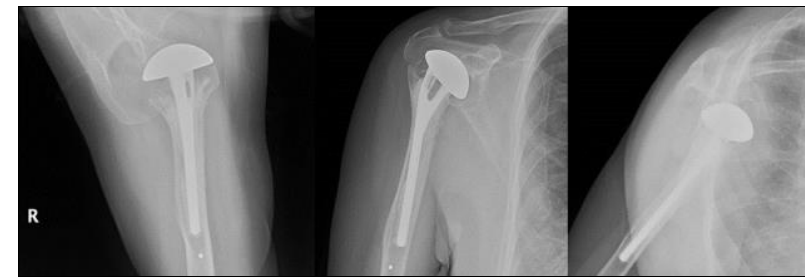

Fig 4: Post-operative radiographs (AP, axillary and scapular Y) of the right shoulder with cemented hemiarthroplasty insitu

Postoperatively the patient was placed in an abduction brace for 4 weeks, then standard sling for 2 weeks. The rehabilitation protocol included passive range of movement within a safe zone (elevation to 90 degrees and external rotation to neutral) for 2 weeks post operatively. The patient then progressed to active assisted movements within the safe zone until week 4 and full active range of movement by week 6 postoperatively.

The patient was reviewed at 2 and 6 weeks, 3 and 6 months (Figure 3). The radiographs showed satisfactory implant position and height with good head size and offset without overstuffing. At 6 months, the patient had no pain (VASp $0 / 10)$, good range of motion (110 degree anterior elevation, 45 degree external rotation and internal rotation up to L3 level) with Subjective Shoulder Value (SSV) of $80 \%$.

\section{Discussion}

The risk of avascular necrosis with fracture dislocations at the anatomical neck of the humerus is significant however, due to their rarity, literature on the outcomes following fixation or hemiarthroplasty is limited ${ }^{[1-9]}$. Silver et al. ${ }^{[2]}$ described one case of a two-part anterior fracture reduced percutaneously with threaded guide pins, however definitive management and its outcomes were not covered as the patient was lost to follow up. Gokkus et al. ${ }^{[1]}$ described a head splitting anterior dislocation managed with open reduction and fixation with 2 kirschner wires and $34 \mathrm{~mm}$ AO cancellous screws, at 15 months follow- up the patient had good pain free function with a Constant shoulder score of 76 points. Several factors may have influenced this favourable outcome. Robinson et al. ${ }^{\text {[3] }}$ presented a 58 patient cohort with anterior fracture-dislocations of the humeral head; though only one case included was a two-part fracture, their conclusions pertain to the particular fracture pattern under discussion. According to Robinson et al. fracture dislocations can be broadly classified as Type 1 or [2]. Type 1 fracture dislocations are less prone to AVN - in Type 1 the humeral head fragment retains its capsular attachments, is over $2 \mathrm{~cm}$ in length and has arterial bleeding. Another indicator of preserved humeral head perfusion is the presence of an extra-articular posteromedial bone spike. In the case described by Gokkus et al. all of these characteristics were present. Further-more the time to surgery was less than 6 hours and evidence suggests late surgery $(>48 \mathrm{~h})$ after injury and poor fracture reduction significantly increase the risk of fixation failure and AVN ${ }^{[5}$, 8]. Primary arthroplasty is recommended in specific 3 or 4 part fractures, as well as head split fractures and those damaging a significant proportion of the articular surface. In cases such as ours, anatomical neck fracture dislocations with large medial calcar spikes, internal fixation would be a recommended first line however hemiarthroplasty is an alternative if there is concerns regarding blood supply and bone quality (as in our case there were poor bone quality and a medial calcar spike without capsular attachment). Hemiarthroplasty allows for early rehabilitation and circumvents the risk of AVN and fixation failure in elderly $[6,10]$.

\section{References}

1. Gokkus K, Agar E, Sagtas E, Aydin AT. Proximal humerus head-splitting fracture with single-part anterior dislocation. BMJ Case Rep, 2014. doi:10.1136/bcr2013-202188

2. Silver MD, Menezes P, Silver JW. Percutaneous Closed Reduction of Fracture-Dislocation of the Shoulder. Orthopedics. 2004; 27(7):713-714.

3. Robinson CM, Khan LA, Akhtar MA. Treatment of anterior fracture-dislocations of the proximal humerus 
by open reduction and internal fixation. $\mathrm{J}$ Bone Joint Surg. Br. 2006; 88:502-8.

4. Siavashi B, Golbakhsh MR. A Case Report of Isolated Posterior Fracture Dislocation of Anatomical Neck of Humerus. Orthop Muscul Syst. 2012; 1:4. DOI: 10.4172/2161-0533.1000116

5. Ide J, Honda K, Takagi K. Posterior dislocation of the shoulder associated with fracture of the humeral anatomical neck with 11 year follow up after early open reduction and internal fixation. Arch Orthop Trauma Surg. 2003; 123:118-120.

6. Compito CA, Self EB, Bigliani LU. Arthroplasty and acute shoulder trauma: reasons for success and failure. Clin Orthop. 1994; 307:27-36.

7. Carofino B, Leopold S. Classifications in Brief: The Neer Classification for Proximal Humerus Fractures. Clin Orthop Relat Res. 2013; 471(1):39-43. DOI: $10.1007 / \mathrm{s} 11999-012-2454-9$

8. Schnetzke M, Bockmeyer J, Loew M, Studier- Fischer S, Grutzner PA, Guehring T. Rate of avascular necrosis after fracture dislocations of the proximal humerus Timing of surgery. Obere Extrem. 2018; 13(4):273278. doi: 10.1007/s11678-018-0452-6

9. Lee CK, Hansen HR. Post-traumatic avascular necrosis of the humeral head in displaced proximal humeral fractures. J Trauma. 1981; 21:788-91.

10. Sperling JW, Cofield RH, Rowland CM. Neer hemiarthroplasty and Neer total shoulder arthroplasty in patients fifty years old or less: longterm results. J Bone Joint Surg Am. 1998; 80-A:464-73. 